\title{
Conditional Deletion of PDK1 in the Forebrain Causes Neuron Loss and Increased Apoptosis during Cortical Development
}

\author{
Congyu $X u^{1}$, Linjie $Y u^{2}$, Jinxing Hou ${ }^{1}$, Rosemary J. Jackson ${ }^{3}$, He Wang ${ }^{1}$, Chaoli Huang ${ }^{1}$, \\ Tingting Liu' ${ }^{1}$, Qihui Wang', Xiaochuan Zou' ${ }^{1}$, Richard G. Morris ${ }^{3,4}$, \\ Tara L. Spires-Jones ${ }^{3,5,6}$, Zhongzhou Yang ${ }^{1}$, Zhenyu Yin ${ }^{7 *}$, Yun $X^{2 *}$ and Guiquan Chen ${ }^{1 *}$ \\ ' State Key Laboratory of Pharmaceutical Biotechnology, Model Animal Research Center, Ministry of Education (MOE) Key \\ Laboratory of Model Animal for Disease Study, Nanjing University, Nanjing, China, ${ }^{2}$ Department of Neurology, Nanjing Drum \\ Tower Hospital, Nanjing University Medical School, Nanjing, China, ${ }^{3}$ Centre for Cognitive and Neural Systems, University of \\ Edinburgh, Edinburgh, United Kingdom, ${ }^{4}$ Consejo Superior de Investigaciones Científicas, Universidad Miguel Hernández, \\ Instituto de Neurociencias, Alicante, Spain, ${ }^{5}$ Centre for Dementia Prevention, University of Edinburgh, Edinburgh, \\ United Kingdom, ${ }^{6}$ Euan MacDonald Centre for Motor Neurone Disease Research, University of Edinburgh, Edinburgh, \\ United Kingdom, ' Department of Geriatrics, Nanjing Drum Tower Hospital, Nanjing University Medical School, Nanjing, \\ China
}

Decreased expression but increased activity of PDK1 has been observed in neurodegenerative disease. To study in vivo function of PDK1 in neuron survival during cortical development, we generate forebrain-specific PDK1 conditional knockout (cKO)

Edited by:

Chao Deng,

University of Wollongong, Australia

Reviewed by:

Jose R. Bayascas,

Universitat Autònoma de Barcelona,

Spain

Yasuhiro Itoh

Harvard University, United States

${ }^{*}$ Correspondence:

Guiquan Chen

chenguiquan@nju.edu.cn

Yun Xu

xuyun20042001@aliyun.com

Zhenyu Yin

zhenyuyin68@163.com

Received: 14 July 2017 Accepted: 05 October 2017 Published: 20 October 2017

Citation:

Xu C, Yu L, Hou J, Jackson RJ, Wang $H$, Huang $C$, Liu T, Wang $Q$, Zou $X$, Morris RG, Spires-Jones $T L$,

Yang Z, Yin Z, Xu Y and Chen G (2017) Conditional Deletion of PDK1 in the Forebrain Causes Neuron Loss and Increased Apoptosis during

Cortical Development

Front. Cell. Neurosci. 11:330. doi: 10.3389/fncel.2017.00330 mice. We demonstrate that $P D K 1 \mathrm{cKO}$ mice display striking neuron loss and increased apoptosis. We report that $P D K 1 \mathrm{cKO}$ mice exhibit deficits on several behavioral tasks. Moreover, PDK1 cKO mice show decreased activities for Akt and mTOR. These results highlight an essential role of endogenous PDK1 in the maintenance of neuronal survival during cortical development.

Keywords: PDK1, Akt, mTOR, neuron loss, apoptosis, learning and memory

\section{INTRODUCTION}

PDK1, a kinase in the PI3K signaling pathway, plays pivotal roles in various biological processes such as cell growth, longevity, and metabolism (Engelman et al., 2006). Activated PI3K phosphorylates phosphatidylinositol bisphosphate $\left(\mathrm{PIP}_{2}\right)$ to generate phosphatidylinositol trisphosphate $\left(\mathrm{PIP}_{3}\right)$. PDK1 is recruited to the intracellular membrane by $\mathrm{PIP}_{3}$ via its $\mathrm{PH}$ domain and then phosphorylates Akt at the 308 threonine (Thr) residue (Alessi et al., 1997; Calleja et al., 2007). On the other hand, the lipid phosphatase PTEN deactivates PDK1 by transforming $\mathrm{PIP}_{3}$ to $\mathrm{PIP}_{2}$ (Engelman et al., 2006). AGC kinase members such as p90 ribosomal S6 kinase 1/2 (RSK1/2), p70 ribosomal S6 kinase (p70S6K), serum- and glucocorticoid-induced protein kinase (SGK), and protein kinase C (PKC) are substrates for PDK1 (Mora et al., 2004; Kharebava et al., 2008).

Due to embryonic lethal effect caused by PDK1 knockout in the whole body, it has been impossible to use $P D K 1^{-/-}$mice to study in vivo functions of PDK1 in the postnatal cerebral cortex. The advantage of viable cell-type specific PDK1 conditional knockout (cKO) mice has helped solve this problem. Recent work showed that conditional deletion of PDK1 through GFAPCre mediated gene recombination causes microcephaly in mice, indicating a critical role of PDK1 in brain development (Chalhoub et al., 2009). A conditional knock-in mouse model expressing the PDK1 L155E mutation displays microcephaly as well (Cordon-Barris et al., 2016). In addition, it 
has been demonstrated that PDK1 in neural progenitor cells (NPCs) is important for the generation of oligodendrocyte precursor cells (Watatani et al., 2012) and neuronal migration (Itoh et al., 2016). The work on epidermis-specific PDK1 cKO mice has revealed an essential role of PDK1 in asymmetric cell division (Dainichi et al., 2016).

Recent evidence has shown that PDK1 is involved in neurodegenerative disease, but how it exerts its role is controversial. On one hand, reduced PDK1 levels (Liu et al., 2011) and impaired PI3K signaling (Steen et al., 2005; Talbot et al., 2012) were found in Alzheimer's disease (AD) brain, suggesting a loss-of-function manner. On the other hand, another group reported that PDK1 activity was increased in $\mathrm{AD}$ brain, and that PDK1 inhibitor enhanced $\alpha$-secretase activity and reduced amyloid plaques in APP transgenic ( $\mathrm{Tg}$ ) mouse models of AD (Pietri et al., 2013), suggesting a gain-of-function mechanism.

Kharebava et al. (2008) have recently demonstrated that over-expression of PDK1 reduces trophic deprivation (TD)induced apoptosis, and that RSK1/2 is required for PDK1mediated neuroprotection. However, it remained uninvestigated whether endogenous PDK1 plays a critical role in neuronal survival during cortical development. We aimed to address this question in the present study. Here, we have crossed $P D K 1^{f / f}$ mice with a forebrain neuron-specific Cre Tg line (Gorski et al., 2002) to generate PDK1 cKO (PDK1 f/f;Emx1Cre) animals, in which PDK1 is inactivated in excitatory neurons of the cortex. We show that PDK1 levels were significantly reduced in the cortex of $P D K 1$ mutant mice. We report that PDK1 cKO mice display striking neuron loss, abnormal apoptosis, and severe memory deficit. We find that $P D K 1 \mathrm{cKO}$ mice exhibit decreased Akt/mTOR activities. These findings highlight a protective role of endogenous PDK1 during brain development.

\section{MATERIALS AND METHODS}

\section{Animal Care and Use}

$P D K 1 \mathrm{cKO}$ mice were generated by crossing floxed $P D K 1^{f / f}$ mice (Feng et al., 2010) with Emx1-Cre Tg (Gorski et al., 2002). Emx1Cre and $m$ TmG mice (Muzumdar et al., 2007) were purchased from the Jackson Laboratory (Bar Harbor, ME, United States). To generate $P D K 1 \mathrm{cKO}$ mice, we crossed homozygous $P D K 1^{f / f}$ with Emx1-Cre to obtain $P D K 1^{f /+} ; E m x 1-C r e$ mice, which were then bred with $P D K 1^{f / f}$ to get $P D K 1^{f / f} ; E m \times 1-C r e$ (PDK1 cKO). $P D K 1^{f /+} ; E m x 1-C r e$ mice grow normally and their brain morphology did not differ from that of $P D K 1^{f /+}$ or $P D K 1^{f / f}$ mice in our analyses. Therefore, $P D K 1^{f /+} ; E m \times 1-C r e$ and $P D K 1^{f / f}$ served as littermate controls to PDK1 cKOs. To detect the floxed $P D K 1$ allele, the following primers were used. The forward primer is TGTGCTTGGTGGATATTGAT and the reverse primer is AAGGAGGAGAGGAGGAATGT.

The mice were kept on 7:00AM-19:00PM light cycle under conditions of constant humidity and temperature $\left(25 \pm 1^{\circ} \mathrm{C}\right)$. The mice were group-housed (4-5 per cage) throughout the experimental period and had ad libitum access to food and water. Both male and female mice were used in this study.
Behavioral experiments were conducted during the light phase of the cycle (8:00AM-18:00PM). Different cohorts of mice were used for behavioral and biochemical experiments. The mice were bred and maintained in an SPF level of animal room in the core facility of the Model Animal Research Center (MARC) at Nanjing University. The genetic background of the mice used in this study was C57BL/6. Mouse breeding was conducted under IACUC approved protocols at the MARC. All the experiments were performed in accordance with the Guide for the Care and Use of Laboratory Animals of the MARC at Nanjing University.

\section{Brain Lysate Preparation}

Mice were euthanized by $\mathrm{CO}_{2}$ at 3 weeks or 3 months of age. Tissues from various brain areas were quickly collected and then placed into liquid nitrogen. Cortical samples were homogenized in cold RIPA (radio immunoprecipitation assay) lysis buffer [consisting of the following (in $\mathrm{mM}$ ): $20 \mathrm{mM}$ Tris-HCl, $\mathrm{pH}$ 7.4, $150 \mathrm{mM} \mathrm{NaCl}, 1 \mathrm{mM}$ EDTA, 1\% NP-40, $0.5 \%$ sodium deoxycholate, and $0.1 \%$ SDS] containing protease and phosphatase inhibitors (Thermo). Lysates were cleared by centrifugation $(14,000 \mathrm{rpm}$ for $15 \mathrm{~min})$. Samples were stored at $-80^{\circ} \mathrm{C}$ until use. Protein concentration was analyzed using a standard BSA method.

\section{Western Blotting}

Normalized cortical samples (40 $\mu \mathrm{g}$ total protein) were used in 12\% SDS-PAGE (Invitrogen) and then transferred to nitrocellulose membrane. After blocking with $5 \%(\mathrm{w} / \mathrm{v})$ dry milk for $1 \mathrm{~h}$, membranes were incubated with primary antibodies overnight and reacted with infrared dye-coupled secondary antibodies (goat anti-rabbit IRdye800, goat antirabbit IRdye680, goat anti-mouse IRdye800, or goat antimouse IRdye680). Membranes were scanned using the Odyssey Infrared Imaging System (Li-Cor). The following antibodies were purchased from the CST: total-Akt (1:1000), pAkt $^{S 473}$ (1:600), GSK $3 \alpha / 3 \beta$ (1:1000), pGSK $3 \alpha^{\mathrm{S} 21} / 3 \beta^{\mathrm{S} 9}$ (1:600), $\mathrm{pAkt}^{\mathrm{T} 308}$ (1:500), pAkt substrates (1:500), pPKA substrates (1:500), pCREB $^{\text {Ser133 }}$ (1:400), pS6K ${ }^{\text {Thr389 }}$ (1:1000), S6K (1:1000), pS6 ${ }^{\text {Ser235/236 }}$ (1:1000), and S6 (1:1000). The following antibodies were also used: antiPDK1 (1:1000, Thermo), PKA Reg1 $\alpha$ (1:1000, Santa Cruz), NeuN (1:500, Millipore), $\beta$-actin (1:10000, Sigma-Aldrich), and GAPDH (1:10000, Genetex).

\section{Paraffin Brain Blocks and Nissl Staining}

The mouse at 3 weeks or 3 months was placed into a $\mathrm{CO}_{2}$ box for about $10 \mathrm{~s}$ so that its respiration became quite faint and its heartbeat got weak. Immediately after the $\mathrm{CO}_{2}$ treatment, cardiac perfusion was conducted using $15 \mathrm{ml} \mathrm{4 \%}$ paraformaldehyde (PFA) solution (in phosphate buffer saline, PBS) in a wellventilated hood. The brain was dissected out and then fixed in PFA overnight. After fixation, the brain was dehydrated and embedded in paraffin.

After embedding, several paraffin brain blocks were prepared. In each block, 4 hemi-brains, including 2 controls and 2 cKOs, were placed together and were sectioned sagittally $(10 \mu \mathrm{m})$ using a microtome (Leica Microsystems, Bannockburn, IL, 
United States). This way allowed 4 brain sections (2 from the control and 2 from the cKO group), which were on identical stereotaxic plane, to be placed on the same slide. For embryos, the head was fixed in 4\% PFA overnight and was later embedded in paraffin. Coronal brain sections for each embryo were collected individually.

Sections were incubated at $58^{\circ} \mathrm{C}$ for $1 \mathrm{~h}$, deparaffinized in xylene and re-hydrated. They were rinsed in PBS for $5 \mathrm{~min}$, soaked in $0.5 \%$ cresyl violet for $12 \mathrm{~min}$, and then dehydrated by a series of ethanol $(70,90,95$, and $100 \%)$. After the sections were cleared in xylene, they were coverslipped with neutral resin (Sinopharm Chemical Reagent Co., Ltd., Shanghai).

\section{The Cortex Volume, Neuron Counting, and Cell Density}

A method described by us previously (Tabuchi et al., 2009; Chen et al., 2010) was used to measure the cortex volume and to count the total number of neurons. It was based on an unbiased stereological neuron counting technique (West and Gundersen, 1990).

The following experiments were conducted to measure the cortex volume for each hemi-brain. First, for each paraffin block, a total of 8 brain slides spaced $400 \mu \mathrm{m}$ apart were selected for Nissl staining. Since each paraffin block contained 2 control and $2 \mathrm{cKO}$ hemi-brains, there were 4 brain sections on each slide. The section thickness was $10 \mu \mathrm{m}$. Second, Nissl-stained images were captured and the area for the cortex in each brain section was measured using the Olympus CellSens Standard system. For each mouse, areas for the cortex from 8 (control) or 6 (cKO) sections were averaged to obtain the mean value across sections. Third, the volume of a hemi-cortex was calculated using the following formula: volume $=$ the mean area $\times$ the total thickness of a hemi-brain. The latter was $3200 \mu \mathrm{m}$ for control but $2400 \mu \mathrm{m}$ for cKO.

The following experiments were performed to calculate the total number of cortical NeuN+ cells for each hemi-brain. First, NeuN immunohistochemistry (IHC) was conducted using 8 slides spaced $400 \mu \mathrm{m}$ apart from each paraffin brain block. Second, for each NeuN-stained section, a total of 10 microscopic fields were randomly selected under the $40 \times$ magnification lens of an Olympus BX53 microscope. Each microscopic field was $100 \mu \mathrm{m} \times 100 \mu \mathrm{m} \times 10 \mu \mathrm{m}\left(10^{5} \mu \mathrm{m}^{3}\right)$ in size and was defined as a counting unit. Third, the total number of NeuN+ cells in each counting unit was counted. The numbers were then averaged across sections to obtain a mean value for $\mathrm{NeuN}+$ cell number per unit. The cell density was defined as the number of NeuN+ cells in a $1 \mathrm{~mm}^{3}$ area. It was calculated using the following formula: density $=$ mean number of NeuN + cell $/ \mathrm{mm}^{3}$. Fourth, the total number of cortical NeuN+ cells in each hemi-brain was calculated using the following formula: total number $=$ cell density $\times$ the cortex volume.

For the measurement of the diameter of cell bodies of NeuN+ cells, a total of 345 cells in the NeuN-stained images for PDK1 cKO at 3 weeks and 365 cells in PDK1 cKO at 3 months were randomly selected to calculate the averaged value $(n=3$ mice/group/age).

\section{BrdU Labeling}

BrdU (B5002, Sigma-Aldrich) was administered to pregnant dams at the concentration of $100 \mathrm{mg} / \mathrm{kg}$. To label proliferating NPCs, BrdU was intraperitoneally injected to pregnant dams at E13.5, E15.5, and E17.5. Embryonic brains were collected $30 \mathrm{~min}$ after the injection and were then processed for paraffin embedding. Each paraffin block contained only one embryonic brain, and serial coronal sections were prepared using a microtome.

\section{Immunohistochemistry and Cell Counting}

Brain sections were immunostained with antibodies against NeuN (1:500, Millipore), PDK1 (1:1000, Abcam), SVP38 (1:500, Millipore), microtubule-associated protein 2 (MAP2) (1:500, Millipore), BrdU (1:500, Abcam), PH3 (1:500, CST), GFAP (1:500, Sigma-Aldrich), and Iba1 (1:500, Wako). For fluorescence IHC (FIHC), sections were incubated with Alexa Fluor 488 secondary antibodies (Invitrogen, 1:500) or Cy3/Cy5 secondary antibodies (Jackson ImmunoResearch; 1:500). Immunofluorescence images were captured using a Leica TCS SP5 laser confocal microscope except for MAP2 staining.

For BrdU+ cell counting, three coronal sections spaced $200 \mu \mathrm{m}$ apart from each embryo were used. Images for cortical BrdU staining were captured under the $40 \times$ objective lens of a Leica confocal laser scanning microscope. In each image, 2 counting units were randomly selected and each unit was an area of $100 \mu \mathrm{m}$ (surface of the ventricular zone, VZ) $\times 200 \mu \mathrm{m}$ (vertical to the VZ surface). The total number of BrdU+ cells in each unit was counted. For each embryo, the number of BrdU+ cells was averaged across 6 counting units to make the mean number.

For PH3+ cell counting, three coronal sections spaced $200 \mu \mathrm{m}$ apart from each embryo were used. Images were captured under the $10 \times$ objective lens of a Leica confocal laser scanning microscope. The total number of $\mathrm{PH} 3+$ cells on the surface of $\mathrm{VZ}$ was counted. The number was then averaged across 3 sections to obtain the mean number of $\mathrm{PH}+$ cells for each embryo.

\section{TUNEL Staining}

Brain sections were blocked with 5\% goat serum for $30 \mathrm{~min}$ and then treated with the TUNEL (terminal deoxynucleotidyl transferase-mediated dUTP-biotin nick end-labeling) BrightGreen Apoptosis Detection Kit (Vazyme) at $37^{\circ} \mathrm{C}$ for $1 \mathrm{~h}$ (Tabuchi et al., 2009). The sections were washed using TBS (Tris-buffered saline) for three times and then scanned using a Leica confocal laser scanning microscope.

TUNEL was also co-stained with NeuN (1:1000, Millipore), Tuj1 (1:1000, SAB), and GFAP (1:500, Sigma-Aldrich). Sections were incubated with Alexa Fluor 488 secondary antibodies (Invitrogen, 1:500) or Cy3/Cy5 secondary antibodies (Jackson ImmunoResearch; 1:500). Images were captured using a ZEISS LSM 880 confocal laser scanning microscope. 


\section{Analysis of Dendritic Length}

Dendrites for neurons in cortical layer $\mathrm{V}$ and hippocampal CA1 were analyzed. Ctip2 antibody was used to label pyramidal neurons in the above brain regions. Double immunostaining on Ctip2/MAP2 was conducted using brain sections at 3 weeks and 3 months. After the FIHC experiments, images doubly positive for Ctip2/MAP2 were examined and observed under the $20 \times$ objective lens of a ZEISS LSM 880 confocal laser scanning microscope. By this way, we could identify Ctip2+ cells displaying long MAP2+ dendrites in cortical layer V. For each mouse, we examined 2-3 brain sections to trace 10 cortical Ctip2+ cells which sent long and unbroken MAP2+ dendrites to cortical layer I/II. In hippocampal CA1, apical dendrites of Ctip2+ cells were quite long and it was relatively easy to get measured. Images were captured under the $10 \times$ objective lens and then processed by Image ${ }^{1}$ to measure dendritic length. For each brain area, a total of 30 Ctip $2+$ neurons from three mice per group were analyzed.

\section{Morris Water Maze Test}

The water maze is a circular pool (1.6 $\mathrm{m}$ in diameter). In the hidden platform task, the platform $(10 \mathrm{~cm}$ in diameter) was kept under water and maintained in the same position. The mice were trained to learn the hidden platform with four trials per day (the inter trial interval, ITI $=15 \mathrm{~min}$ ) for 5 days. Each training trial lasted for $60 \mathrm{~s}(\mathrm{~s})$. If the mice were unable to find the platform, they were guided to it by hand and were allowed to stay on it for $30 \mathrm{~s}$. The swimming path of the mice was monitored using the ANY-Maze ${ }^{\circledR}$ tracking system (ANY-Maze, Stoelting Co., Wood Dale, IL, United States). After the last training trial of $24 \mathrm{~h}$ on day 5 , the mice were subjected to a probe test in which the platform was removed and the mice were allowed to search for it for $60 \mathrm{~s}$.

\section{Rotarod Test}

The rotarod test was conducted in the same experimental room for the open-field test. The mice were placed in a neutral position on a stationary rotarod $(3 \mathrm{~cm}$ in diameter, ShangHai Biowill Co., Ltd., Shanghai). Timers were used to record the time to fall. Mice were tested on the rotarod at constant rotation speeds of 10,20 , 30 , and $40 \mathrm{rpm} / \mathrm{min}$.

\section{Open-Field Test}

The ANY-Maze ${ }^{\circledR}$ system was used to monitor locomotion of the mice. A $40 \mathrm{~cm} \times 40 \mathrm{~cm}$ plexiglas chamber was set in a quiet laboratory room. The area, $10 \mathrm{~cm}$ to the walls, was defined as the wall area. During the test, the mouse was placed in the center of the open-field chamber and allowed to move for $10 \mathrm{~min}$. After each testing trial, the chambers were thoroughly cleaned by $70 \%$ ethanol to get rid of odors left by animals tested in a previous trial. The total distance traveled and the time spent in different areas were recorded.

\section{Data Analysis}

Data were presented as the mean \pm SEM. For behavioral data and cell counting data, analysis of variance (ANOVA) was conducted

\footnotetext{
${ }^{1}$ https://imagej.nih.gov/ij/
}

to compare main genotype effects. For biochemical results, twotailed Student's $t$-test was performed to examine the difference between control and cKO mice; $p<0.05\left(^{*}\right)$ and $p<0.01\left(^{* *}\right)$ were considered statistically significant and highly significant, respectively.

\section{RESULTS}

\section{Reduced Size of the Cortex in PDK1 cKO Mice}

Early work has shown that $P D K 1^{-/-}$mice display multiple abnormalities and die at embryonic day 9.5 (E9.5) (Lawlor et al., 2002). In this study, we have generated viable forebrain-specific PDK1 cKO $\left(P D K 1^{f / f} ; E m \times 1-C r e\right)$ mice. These mutant animals exhibited smaller cerebrum than controls did (Figure 1A). To examine the inactivation pattern of PDK1 mediated by Cre recombination, the $m T m G$ mouse (Muzumdar et al., 2007) was crossed to the Emxl-Cre mouse to obtain EmxlCre; $m T m G$. In the latter, the expression of green fluorescence protein (GFP) was mainly observed in the cortex and the hippocampus (Figure 1B). To examine the inactivation efficiency of PDK1, we performed Western blotting for PDK1 using cortical homogenates. Significantly reduced PDK1 protein levels were observed in the cortex of PDK1 cKO mice at 3 weeks and 3 months of age (Figure 1C). The residual amount of PDK1 was likely from cells including GABAergic neurons, blood cells, glial cells, and a small proportion of excitatory neurons that do not express the Cre recombinase. We further conducted double-immunostaining for $\mathrm{NeuN}$ and PDK1. We found that the majority of cortical NeuN positive (+) cells in PDK1 cKO mice were PDK1 negative. In contrast, neurons in control mice were doubly positive for NeuN/PDK1 (Figure 1D).

Nissl staining was used to examine brain morphology. There was remarkable reduction on the size of the cortex in PDK1 cKO mice (Figure 1E). The thickness of the cortex in PDK1 cKO mice was decreased by about $50 \%$ as compared to age-matched littermate controls (Figure 1Ea'-d'). Quantification data showed that the cortical volume was dramatically decreased in PDK1 cKO mice either at 3 weeks or 3 months (Figure 1F). In contrast, the size of the cerebellum in PDK1 cKO mice was not decreased at either age (Figure 1E), likely due to that Cre recombinase was not expressed in cerebellar neurons. Moreover, the measurement on the averaged area of the cerebellum per section showed no significant difference between two genotypes at either age ( 3 weeks: control $=100 \pm 2.6 \%, \mathrm{cKO}=103.1 \pm 2.3 \% ; 3$ months: control $=100 \pm 3.5 \%, c K O=99.4 \pm 3.7 \%$; ps > 0.6, Student's $t$-test).

\section{Loss of Mature Neurons in PDK1 cKO Mice}

We measured the number of mature neurons using NeuN as a marker. First, IHC on NeuN revealed significant reductions in the cortex size and the total number of NeuN+ cells in PDK1 cKO mice aged at 3 weeks and 3 months (Figure 2A). Consistent with this, Western analysis confirmed reduced NeuN levels 

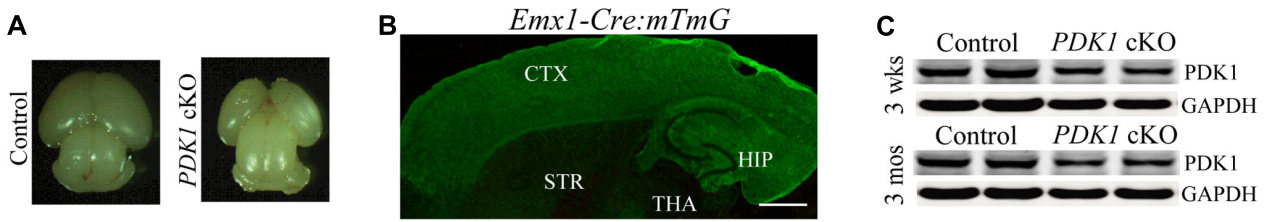

D
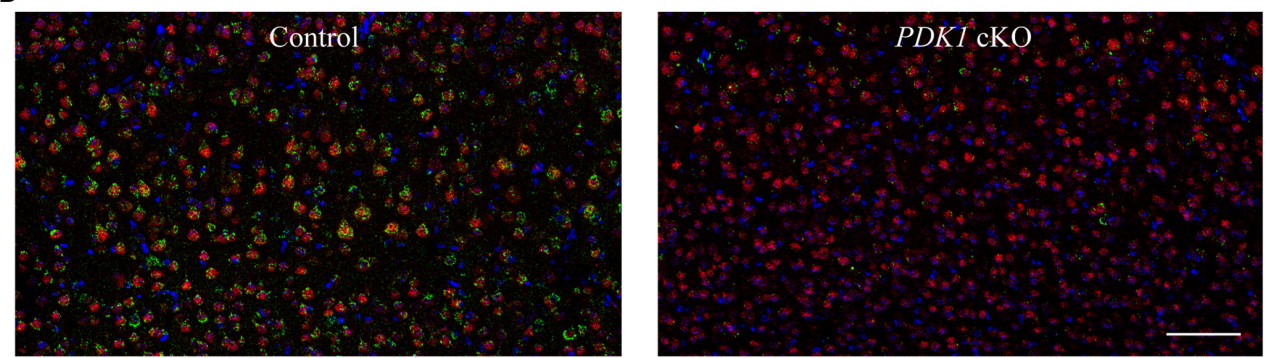

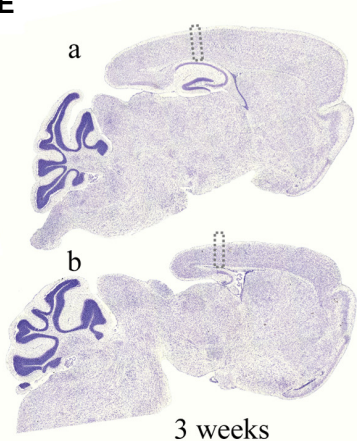

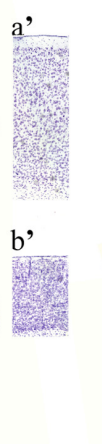
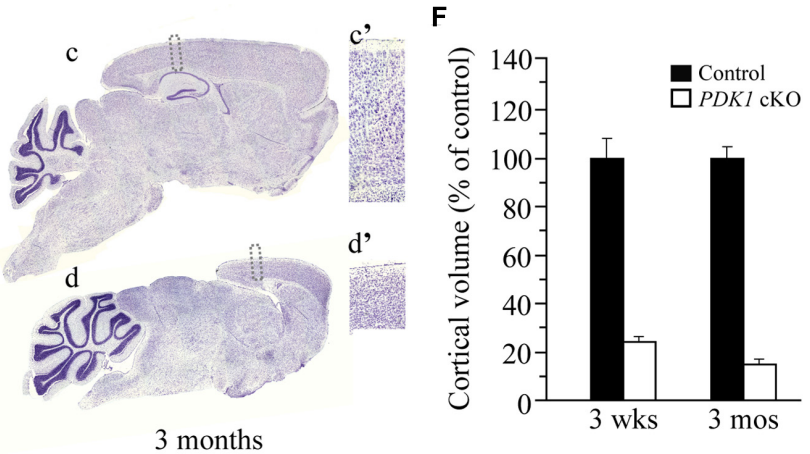

FIGURE 1 | Reduced size of the cortex in forebrain-specific PDK1 cKO mice. (A) Representative brain photos for a control mouse and a PDK1 cKO mouse at P0. (B) The expression pattern of GFP in the brain of a 3-week-old Emx1-Cre;mTmG mouse. GFP was mainly expressed in the cortex (CTX) and the hippocampus (HIP) but not the striatum (STR), the thalamus (THA), or the cerebellum. (C) Western analysis on PDK1. Cortical homogenates from mice aged at 3 weeks and 3 months ( $n=3-4$ /group/age) were prepared. There was significant difference on protein levels of PDK1 between control and $P D K 1 \mathrm{cKO}$ mice $(3 \mathrm{weeks}$ : control $=100 \pm 4.6 \%$, $\mathrm{cKO}=58.0 \pm 5.8 \%$; 3 months: control $=100 \pm 3.5 \%$, cKO = 60.3 $\pm 2.1 \%$; ps < 0.005). GAPDH served as the loading control. (D) Double-immunostaining for NeuN/PDK1 in mice at 3 weeks of age. Most NeuN+ cells in control brain were PDK1+ but very few NeuN+ cells in PDK1 cKO brain were PDK1+. Scale bar $=100 \mu \mathrm{m}$. (E) Nissl staining for mice at 3 weeks and 3 months. Boxed areas in control (a,c) and cKO (b,d) were enlarged as a', c', b', and d', respectively. (F) Relative cortical volume. There was significant difference on the size of the cortex between control and PDK1 cKO mice at 3 weeks (control = $100 \pm 9.9 \%$, $\mathrm{cKO}=24.1 \pm 2.0 \% ; n=3-4$ mice/group; $p<0.001$ ) or 3 months (control = $100 \pm 4.4 \%$, cKO = 14.9 $\pm 1.9 \% ; n=3-4$ mice/group; $p<0.001$ ).

(Figure 2B). Second, a stereological cell counting method was used to count NeuN+ cell number (West and Gundersen, 1990; Tabuchi et al., 2009; Chen et al., 2010). Our results showed that the total number of NeuN+ cells was dramatically decreased in $P D K 1 \mathrm{cKO}$ mice (Figure 2C). The measurement on the diameter for the cell body of NeuN+ cells indicated smaller size of neurons in PDK1 cKO mice than in controls (Figure 2D). However, the density of NeuN+ cells in PDK1 cKO mice was increased as compared to control animals (Figure 2E). This finding was in agreement with that reported on PDK1 conditional knock-in mice (Cordon-Barris et al., 2016). Overall, conditional deletion of PDK1 in the forebrain resulted in remarkable loss of mature neurons.

To investigate whether neuron loss occurred via apoptosis, we conducted the TUNEL assay (Tabuchi et al., 2009). Brain sections of PDK1 cKO mice aged at P0, 1 week, and 3 weeks were used. We found that the total number of TUNEL + cells in the cortex of PDK1 mutants was increased (Figure $2 \mathrm{~F}$ and
Supplementary Figure 1). Cell counting results confirmed that the averaged number of TUNEL+ cells per section in PDK1 cKO mice was significantly larger than that in controls (Figure 2G: $F=60, \mathrm{df1} / 8, p<0.001$ and Supplementary Figure 1), suggesting enhanced apoptotic cell death. To identify which cell type underwent apoptosis, we performed double-staining for TUNEL/NeuN, TUNEL/Tuj1, or TUNEL/GFAP (Figure $\mathbf{2 H}$ ). Only TUNEL+/NeuN+ and TUNEL+/Tuj1+ cells (Figure $\mathbf{2 H}$, indicated by white arrows) were observed in $P D K 1 \mathrm{cKO}$ mice as compared to controls. No TUNEL+/GFAP+ cells were detected (Figure 2H).

\section{Unchanged Self-renewal of NPCs in PDK1 cKO Mice}

To determine the possibility that loss of cortical neurons was caused by deficient self-renewal of NPCs, we first performed BrdU pulse-labeling experiment, in which BrdU labels NPCs at the S-phase of the cell cycle. No qualitative changes on 

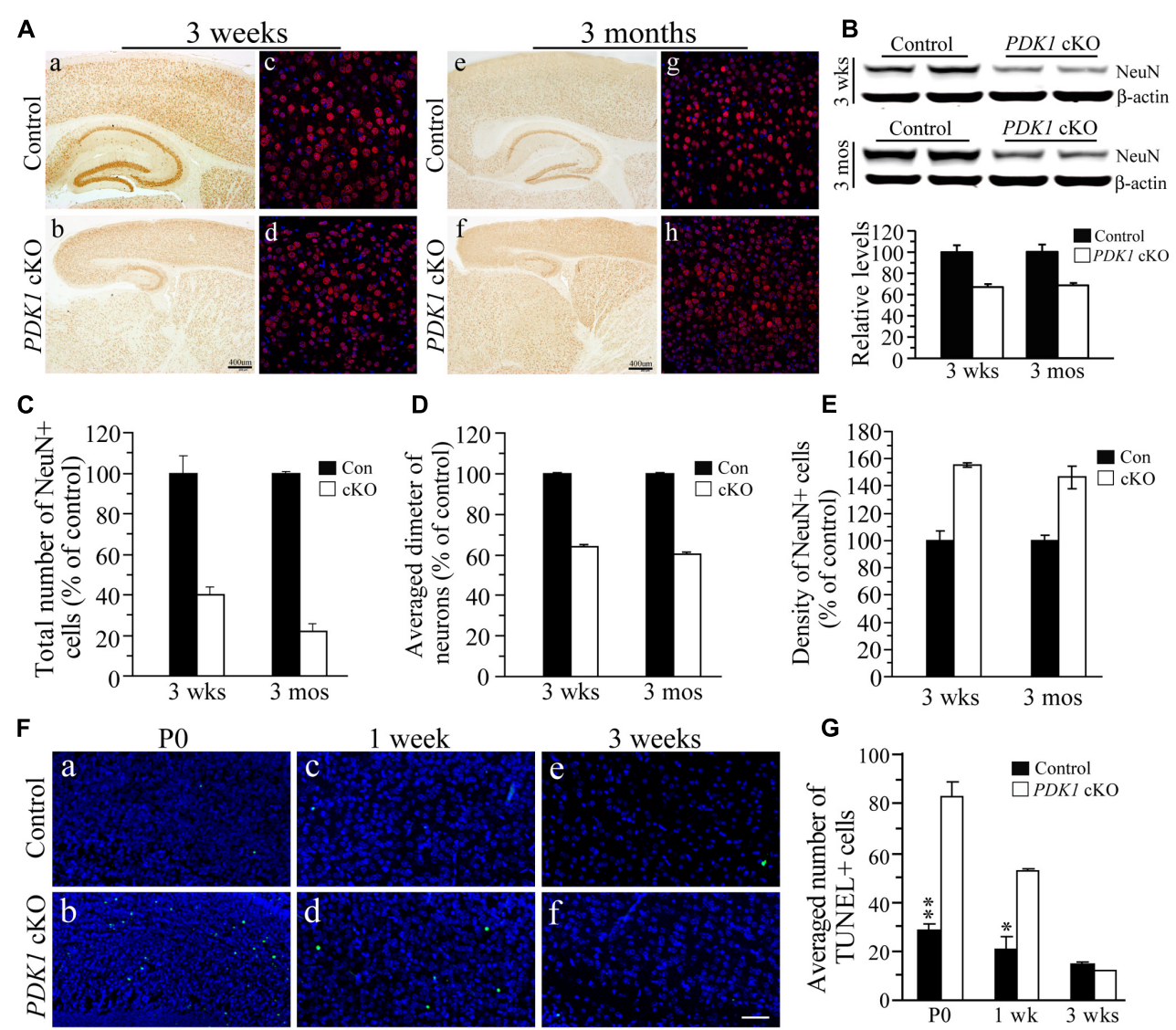

\section{D}

E
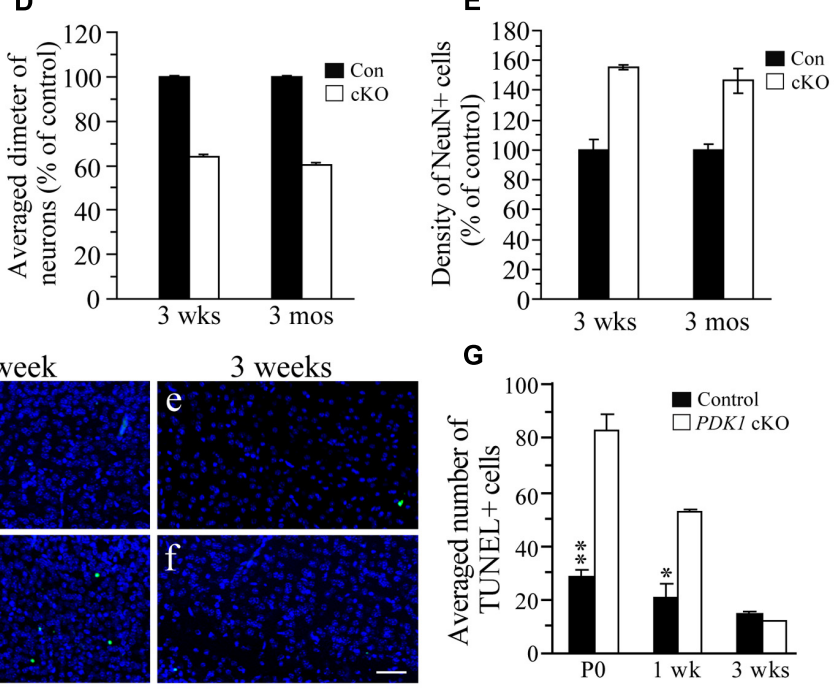

H

TUNEL

NeuN

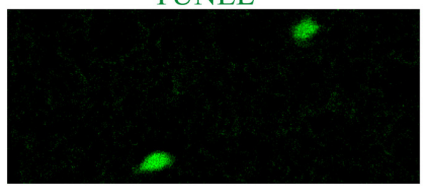

TUNEL

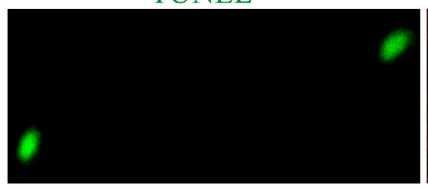

TUNEL

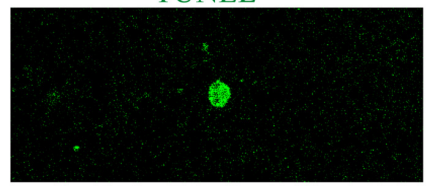

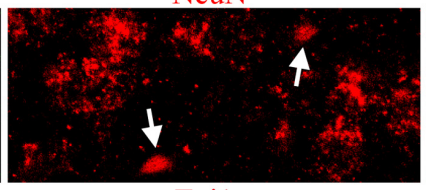

Tuj1

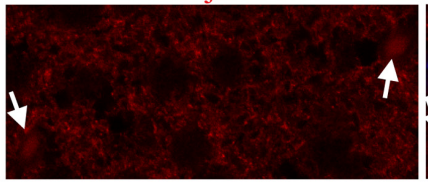

GFAP

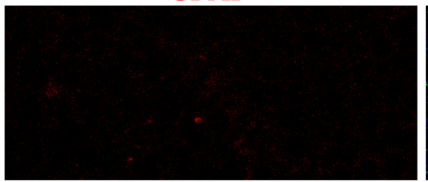

TUNEL/NeuN

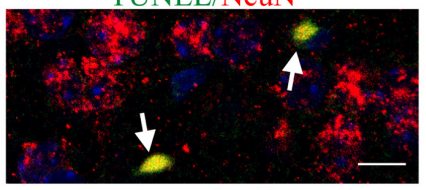

TUNEL/Tuj1

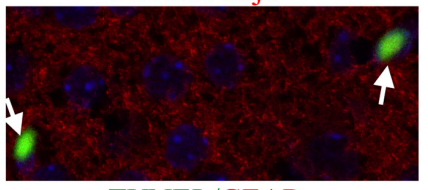

TUNEL/GFAP

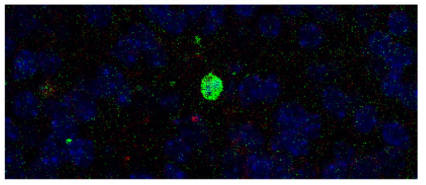

FIGURE 2 | Remarkable loss of mature neurons in PDK1 cKO mice. (A) $1 \mathrm{HC}$ for NeuN. There were significant reductions on NeuN+ cells in the cortex and the hippocampus of $P D K 1 \mathrm{cKO}$ mice at 3 weeks (a-d) and 3 months (e-h). Scale bar $=50 \mu \mathrm{m}$. (B) Western blotting on NeuN. There were significant differences on NeuN protein levels between control and PDK1 cKO groups at 3 weeks and 3 months ( $p s<0.01$ ). $\beta$-actin served as the loading control. (C) Relative number of $\mathrm{NeuN}+$ cells was significantly different between control and PDK1 cKO mice at 3 weeks (control $=100 \pm 9.8 \%, \mathrm{cKO}=39.8 \pm 3.8 \% ; n=3-4 / \mathrm{group} ; p<0.005$ ) or 3 months (control $=100 \pm 0.8 \%, \mathrm{cKO}=22.0 \pm 3.6 \% ; n=3-4$ /group; $p<0.001$ ). (D) Relative diameter of NeuN+cells was significantly decreased in PDK1 cKO mice at 3 weeks (control $=100 \pm 0.2 \%, n=118$ cells from 3 mice; $\mathrm{cKO}=60.5 \pm 0.1 \%, n=345$ cells from 3 mice; $p<0.001$ ) or 3 months (control $=100 \pm 0.2 \%$, $n=110$ cells from 3 mice; $c K O=64.1 \pm 0.2 \%, n=365$ cells from 3 mice; $p<0.001$ ). (E) Cell density of NeuN+ neurons (per $1 \mathrm{~mm}^{3}$ area) was significantly increased in PDK1 cKO mice at 3 weeks (control $=100 \pm 3.9 \%, \mathrm{CKO}=146.3 \pm 8.4 \% ; n=4$ mice/group; $p<0.01$ ) or 3 months (control $=100 \pm 7.2 \%$, $\mathrm{CKO}=155.5 \pm 1.7 \%, n=4$ mice/group; $p<0.001)$. (F) TUNEL staining in the cortex of the mice aged at PO (a,b), 1 week (c,d), and 3 weeks (e,f). Scale bar $=50 \mu \mathrm{m}$. (G) The averaged number of TUNEL+ cells per section. There were significant increases in PDK1 cKO mice at PO and 1 week but not 3 weeks $\left({ }^{*} p<0.05 ;{ }^{* *} p<0.01\right)$. (H) Double-immunostaining for TUNEL/NeuN, TUNEL/Tuj1, or TUNEL/GFAP. Cells doubly positive for TUNEL(green)/NeuN(red) or TUNEL(green)/Tuj1 (red) were indicated by white arrows in PDK1 cKO mice. Cells doubly positive for TUNEL(green)/GFAP(red) were not found. Scale bar $=10 \mu \mathrm{m}$. 
BrdU immunoreactivity were detected in PDK1 cKO mice at embryonic day 13.5 (E13.5), E15.5 or E17.5, as compared to age-matched controls (Supplementary Figure 2A). Cell counting results showed no significant reduction in the averaged number of BrdU+ cells per $100 \mu \mathrm{m} \times 200 \mu \mathrm{m}$ area of the VZ/SVZ (sub$\mathrm{VZ}$ ) in PDK1 cKO embryos (Supplementary Figure 2B: $p>0.2$ for each age).

We next conducted FIHC for PH3, a marker for NPCs at the $\mathrm{M}$-phase of the cell cycle. There was no qualitative difference in the immunoreactivity of $\mathrm{PH} 3$ between control and PDK1 cKO embryos (Supplementary Figure 2C). The averaged number of
$\mathrm{PH} 3+$ cells in the surface of the VZ for PDK1 cKO embryos was not significantly decreased (Supplementary Figure 2D: $p>0.2$ for each age). Overall, the self-renewal or proliferation of NPCs was not impaired in $P D K 1 \mathrm{cKO}$ mice.

\section{Loss of Synapses and Dendrites in PDK1 cKO Mice}

To study whether the morphology of synapses and dendrites was affected in PDK1 cKO mice, we first conducted FIHC on synaptophysin (SVP38), a marker for pre-synaptic element. Qualitatively reduced intensity of SVP38 immunoreactivity was
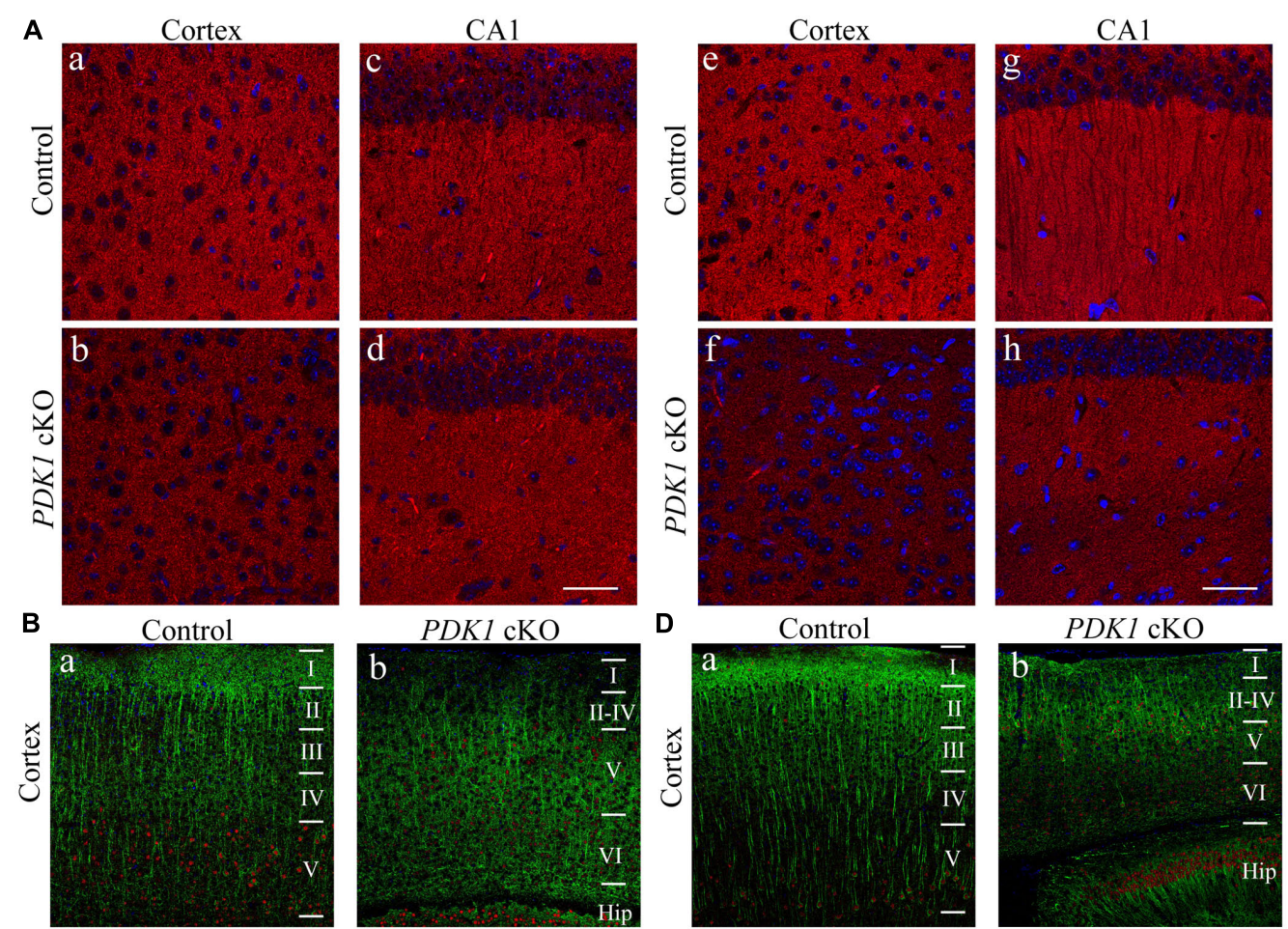
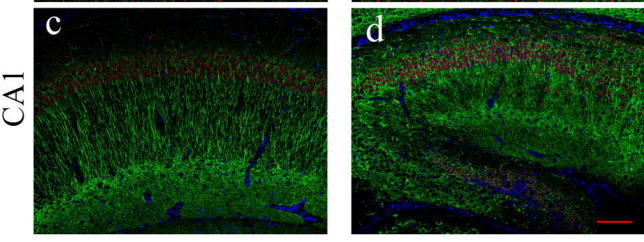

C

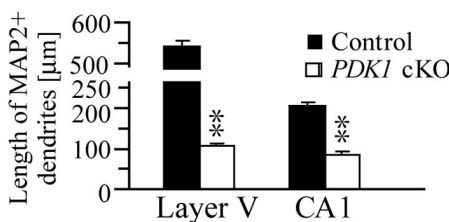

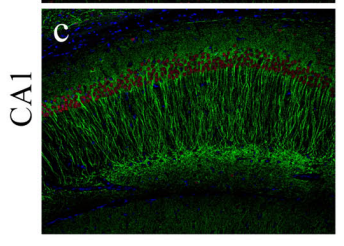
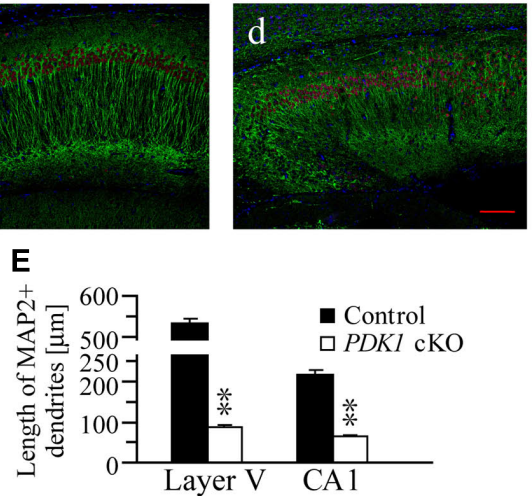

FIGURE 3 | Loss of synapses and dendrites in PDK1 CKO mice. (A) IHC for SVP38. Qualitatively altered immunoreactivity of SVP38 in the cortex and the hippocampus in PDK1 cKO mice aged at 3 weeks (a-d) or 3 months (e-h). Scale bar = $100 \mu \mathrm{m}$. (B) Double-staining of MAP2/Ctip2 in the cortex (a,b) and the hippocampus (c,d) of mice at 3 weeks. Dendrites, neurons, and cell bodies were labeled by MAP2 (green), Ctip2 (red), and DAPI (blue), respectively. Scale bar $=100 \mu \mathrm{m}$. (C) There were significant differences on the averaged dendritic length for neurons in layer $\mathrm{V}$ of the cortex and in hippocampal CA1 area of control and PDK1 cKO mice (30 Ctip2+ neurons from 3 mice per brain area per group; $\left.{ }^{* *} p<0.01\right)$. (D) Double-staining of MAP2/Ctip2 in mice at 3 months (a-d). Scale bar $=100 \mu \mathrm{m}$. (E) The averaged length for dendrites was significantly different between two groups (30 Ctip2+ neurons from 3 mice per brain area per group; $\left.{ }^{* *} p<0.01\right)$. 
A

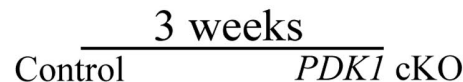

a

b

B

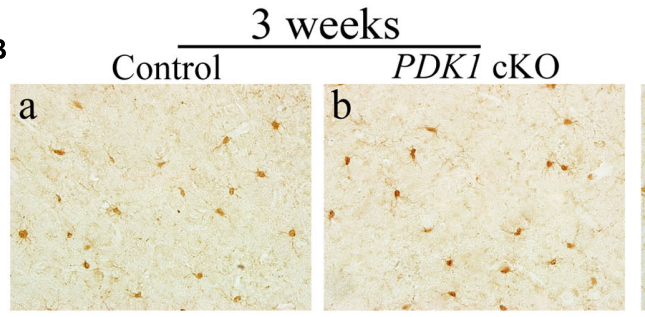

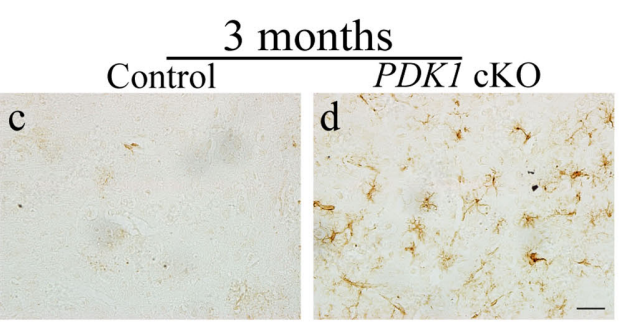

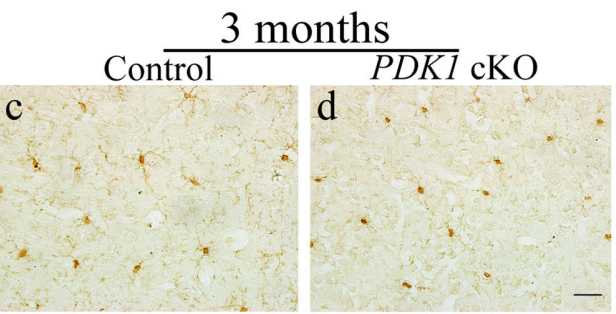

C

GFAP
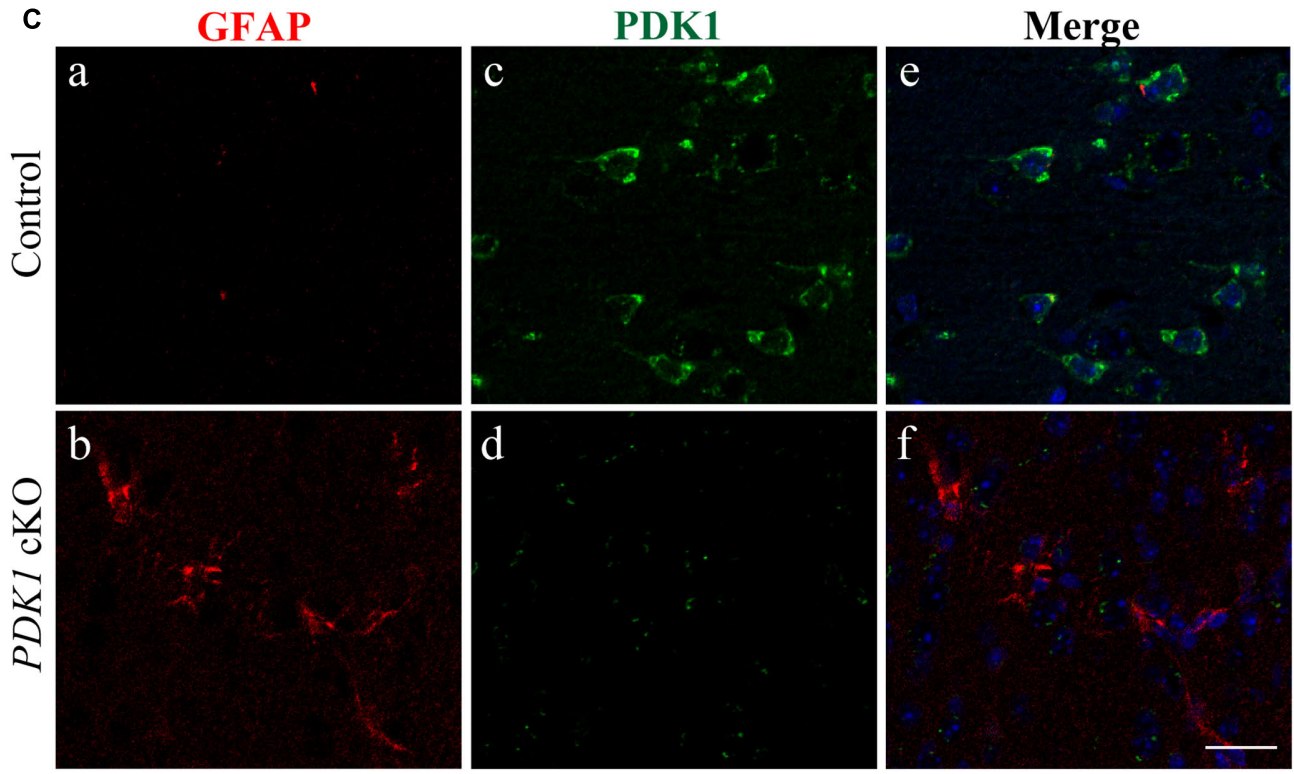

FIGURE 4 | Astroglial activation in PDK1 cKO mice. (A) IHC for GFAP. There was increased number of GFAP+ cells in the cortex of PDK 1 cKO mice at 3 weeks or 3 months. Scale bar $=20 \mu \mathrm{m}$. (B) $\mathrm{HC}$ for lba1. There was no significant change on the total number of lba1+cells in the cortex of $P D K 1 \mathrm{cKO}$ mice at $3 \mathrm{weeks}$ or 3 months. Scale bar $=20 \mu \mathrm{m}$. (C) Double-immunostaining for GFAP/PDK1. Abundant PDK1+ neurons but few GFAP+ cells were seen in the cortex of control mice (a,c,e). GFAP+ cells were PDK1 negative in PDK1 cKO mice (b,d,f). Scale bar $=25 \mu \mathrm{m}$.

observed in the cortex and the hippocampus of PDK1 cKO mice across ages (Figure 3A), suggesting loss of synapses. In addition, Western analyses on SVP38 and post-synaptic density 95 (PSD95) showed that their levels were significantly decreased in PDK1 cKO mice (data not shown). We then performed double-immunostaining of MAP2/Ctip2, markers for dendrites and pyramidal neurons in cortical layer $\mathrm{V}$, respectively. We found that the immunoreactivity of MAP2 was qualitatively reduced in the cortex of PDK1 cKO mice as compared to age-matched littermate controls (Figures 3B,D), suggesting loss of dendrites. We further measured the length of dendrites of Ctip2+ neurons. The averaged dendritic length was significantly decreased in PDK1 cKO mice either at 3 weeks (Figure 3C) or 3 months
(Figure 3E). For neurons in cortical layer V, there was more than $80 \%$ of reduction on the averaged dendritic length in PDK1 mutants. For pyramidal neurons in hippocampal CA1, the averaged dendritic length was also dramatically reduced in $P D K 1$ cKO mice (Figures 3C,E).

\section{Astrocytosis in PDK1 cKO Mice}

To study whether there were changes on glial cells in the cortex of PDK1 cKO mice, first, IHC on GFAP was performed. Increased number for GFAP + cells was observed in the cortex of PDK1 cKO mice. The increase in the immunoreactivity of GFAP was subtle in PDK1 cKOs at 3 weeks (Figure 4Aa,b) but more robust at 3 months (Figure $4 \mathrm{Ac}, \mathrm{d}$ ), suggesting progressive 

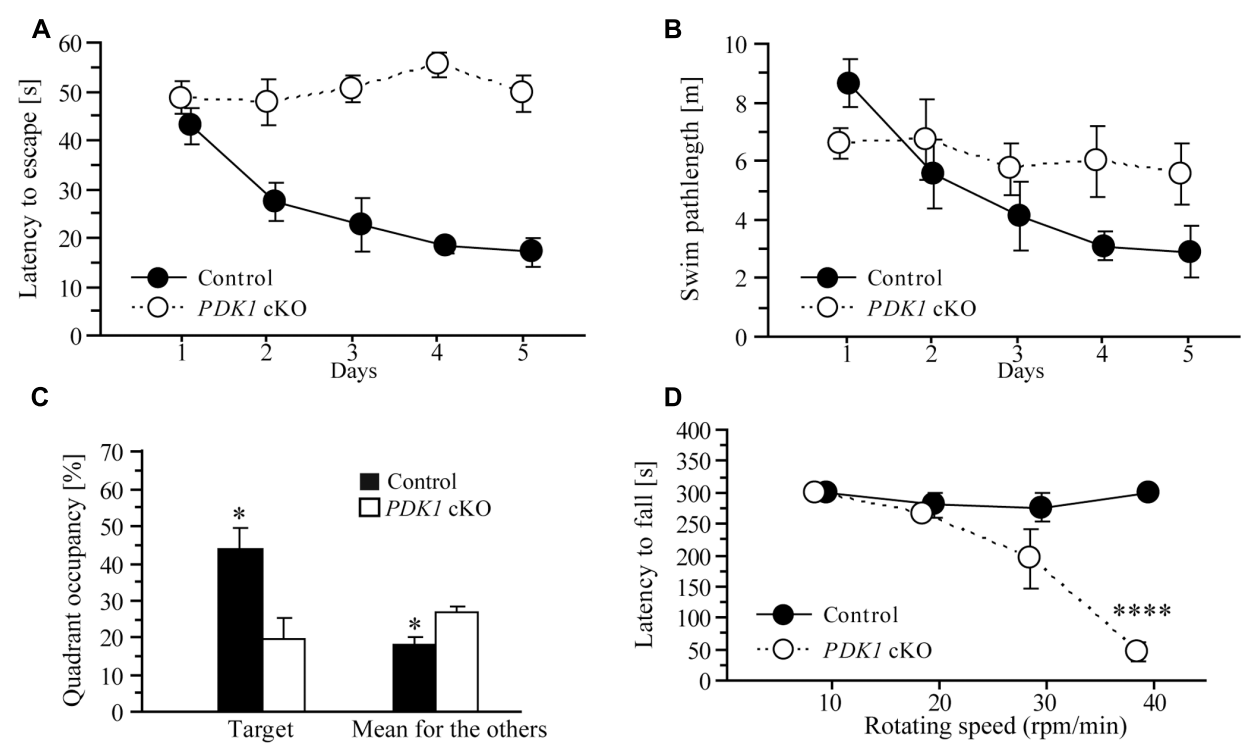

FIGURE 5 | Learning deficit in PDK1 cKO mice. (A) Escape latency for control and PDK1 cKO mice which were trained with a hidden platform task for 5 consecutive days. There was significant difference on the latency to escape between the two genotype groups ( $n=6 /$ group). PDK 1 cKO mice showed no improvement on their performance during the 5-day training. (B) The length of swim-path. There was significant difference between control and PDK1 cKO mice across the training period. (C) Quadrant occupancy in a probe test conducted $24 \mathrm{~h}$ after the last training trial. There was significant difference on the time spent in the target quadrant between control and PDK1 cKO mice $\left({ }^{*} p<0.05\right)$. There was also significant difference in the average time spent in the remaining three quadrants (adjacent left, adjacent right, and opposite) between control and PDK1 cKO animals ( $\left.{ }^{*} p<0.05\right)$. (D) Latency to fall from a rotarod. Four different rotating speeds including 10, 20, 30, and $40 \mathrm{rpm} / \mathrm{min}$ were used. There was no significant difference on the latency to fall between control $(n=10)$ and $P D K 1 \mathrm{cKO}$ mice $(n=6)$ at lower speeds $(p s>0.1)$. There was significant difference between control and PDK1 cKO mice at the highest speed $(* * * *<0.001)$.

astroglial activation. Second, IHC on Iba1 was conducted. The immunoreactivity of Iba1 in control and PDK1 cKO mice did not qualitatively differ at either age (Figure 4B), indicating no significant microgliosis.

Since the Cre recombinase was also expressed in astrocytes in $P D K 1^{f / f}$;Cre mice, this suggested a possibility that loss of PDK1 may affect the cell number of astrocytes. We conducted doubleimmunostaining for GFAP/PDK1. We found that GFAP+ cells were largely PDK1 negative in $P D K 1 \mathrm{cKO}$ mice (Figure 4C), indicating that PDK1 was inactivated in astrocytes. Moreover, it has been demonstrated that astrocytosis is associated with neuron loss in neurodegenerative mouse models (Saura et al., 2004; Tabuchi et al., 2009; Cheng et al., 2015). Overall, astroglial activation in $P D K 1 \mathrm{cKO}$ mice may be due to neuronal death and a cell autonomous function of PDK1 in astrocytes.

\section{Learning Deficit in PDK1 cKO Mice}

To determine whether cognitive ability of PDK1 mutant mice was affected, we used a Morris water maze task to test spatial learning. In this task, 2- to 3-month-old mice were trained to learn a hidden platform for 5 days. During the 5-day training period, there was no improvement on the latency to escape in PDK1 cKO mice. ANOVA confirmed a highly significant main genotype effect $(F=46.1, d f=1 / 10, p<0.001)$ between two groups of mice across 5 days (Figure 5A). There was also significant genotype effect $(F=11.6, d f=3.0 / 30.1, p<0.001)$ on the length of swim-path (Figure 5B). Overall, these results indicated that spatial learning was severely affected in PDK1 cKO mice.
One day after the last training trial, the mice were subjected to a probe test, in which no platform was available in the water. The average time spent in the target and other quadrants of the water maze was analyzed (Figure 5C). Significant genotype effect $(F=8.5, d f=1 / 10, p<0.05)$ and significant quadrant $\times$ genotype effect $(F=4.0, d f=2.4 / 23.9, p<0.05$, Greenhouse-Geisser correction) were observed, suggesting that no spatial memory had formed in PDK1 cKO mice. We also found that the control mouse preferred to search for the target quadrant but the $P D K 1 \mathrm{cKO}$ swam randomly during the probe test (data not shown).

Next, a rotarod task was conducted to examine motor learning. A number of different rotating speeds including 10, 20, 30, and $40 \mathrm{rpms} / \mathrm{min}$ were used. The latency to fall from the rotating rod was averaged (Figure 5D). ANOVA revealed a significant speed $\times$ genotype effect $(F=22.8, d f=1.5 / 21.0$, $p<0.001$ ). At lower speeds including 10,20 , or $30 \mathrm{rpm} / \mathrm{min}$, there were no significant genotype effects on the latency to fall between control and PDK1 cKO mice ( $p$ s $>0.1$ ). However, there was significant genotype effect at the speed of $40 \mathrm{rpm} / \mathrm{min}(p<0.001)$, likely suggesting impairment on motor learning.

\section{Decreased Activities for Akt and mTORC1 in PDK1 cKO Mice}

Our biochemical analysis showed that levels of total Akt (T-Akt) were not changed in PDK1 cKO mice at either age (Figure 6A, $p s>0.2$ ), indicating unaltered Akt expression. In contrast, relative levels of $\mathrm{pAkt}^{\mathrm{Thr} 308}$ were decreased in PDK1 
A
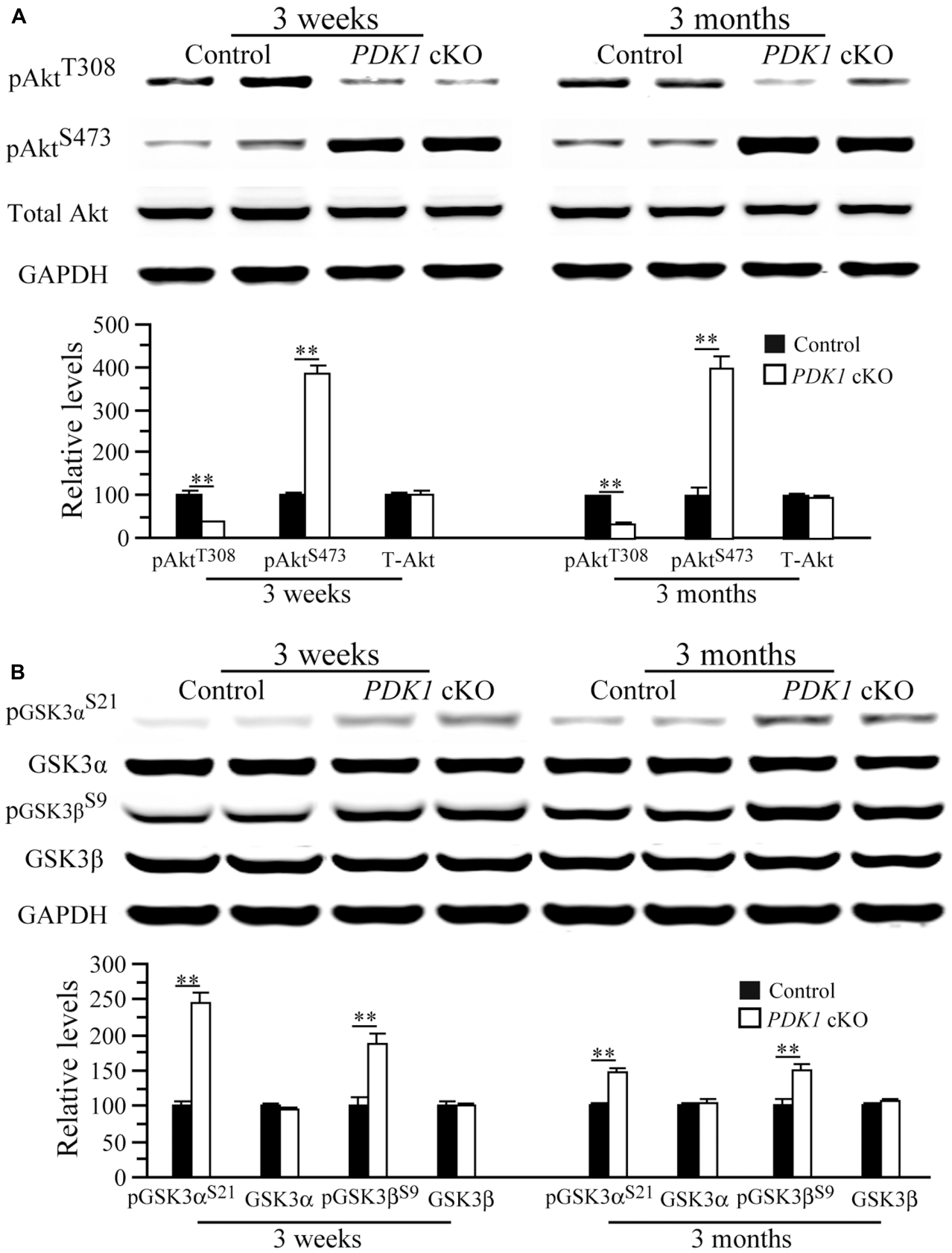

FIGURE 6 | Levels of pAkt and pGSK3 in PDK1 cKO mice. (A) Western analyses on pAkt and total Akt (T-Akt). There were significant differences on relative levels of pAkt ${ }^{\mathrm{T} 308}$ and $\mathrm{pAkt}{ }^{\mathrm{S} 73}$ between control and PDK1 cKO mice at 3 weeks and 3 months ${ }^{* *} p<0.01 ; n=3-4 /$ group/age). There was no significant difference on T-Akt levels between control and PDK1 cKO mice at either age (ps > 0.2). GAPDH served as the loading control. (B) Western analyses on pGSK3 $\alpha$, pGSK3 $\beta$, total GSK3 $\alpha$, and total GSK3 $\beta$. There were significant differences on relative levels of pGSK3 $\alpha^{S 21}$ and pGSK3 $\beta^{S 9}$ between control and PDK1 cKO mice at 3 weeks and 3 months $\left({ }^{* *} p<0.01\right)$. There were no significant differences on levels of total GSK3 $\alpha$ and GSK3 $\beta$ between control and PDK1 cKO mice (ps > 0.5). GAPDH served as the loading control.

cKO mice (Figure 6A) (3 weeks: control $=100 \pm 10.5 \%$, $\mathrm{cKO}=37.0 \pm 1.1 \% ; 3$ months: control $=100 \pm 1.2 \%$, $\mathrm{cKO}=35.5 \pm 1.3 \% ; n=3$-4/group/age; ps $<0.01)$. In contrast, levels of $\mathrm{pAkt}^{\mathrm{Ser} 473}$ were increased in PDK1 cKO mice (Figure 6A, $p s<0.01$ ). In agreement with this, increased levels of $\mathrm{pAkt}^{\mathrm{Ser} 473}$ were reported in the brain of $P D K 1^{f / f}$;GFAP-Cre mice (Figure 4 in Chalhoub et al., 2009).

The finding of reduced $\mathrm{pAkt}^{\mathrm{Th} 308}$ levels suggested that Akt activity might be decreased. As a major Akt substrate, GSK3 was then examined. Levels of total GSK $3 \alpha$ and GSK3 $\beta$ did not differ between two groups (Figure 6B), but those for pGSK $3 \alpha^{\text {Ser21 }}$ 

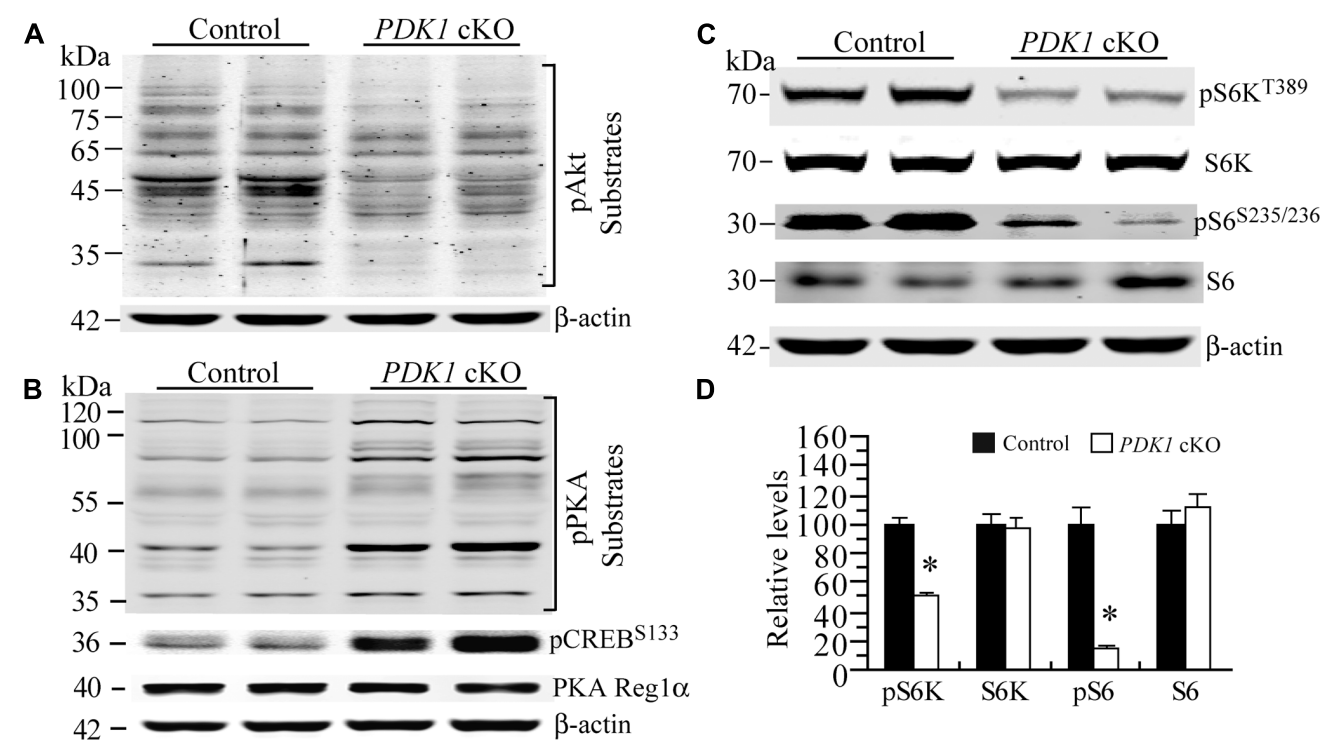

D

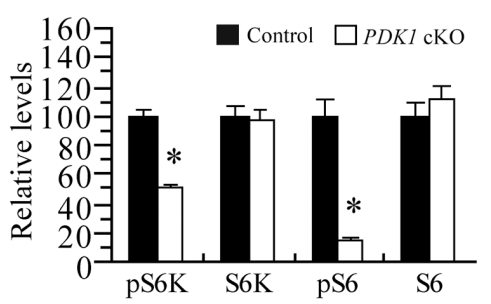

FIGURE 7 | Enhanced PKA activity but decreased mTORC1 activity in PDK1 cKO mice. (A) Western blotting for pAkt substrates. Several bands for Akt downstream targets were decreased in PDK1 cKO mice ( $n=3$ mice/group). (B) Western blotting for pPKA substrates, pCREB and PKA Reg1 $\alpha$. Several PKA downstream targets

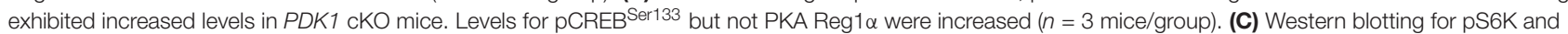
pS6 in control and PDK1 cKO mice. (D) There were significant differences on relative levels of pS6K and pS6 between control and PDK1 cKO mice at 3 months $\left(n=3\right.$ mice/group; $\left.{ }^{*} p<0.05\right)$. There were no significant differences on levels of total S6K and S6 between control and PDK1 cKO mice (ps $\left.>0.5\right)$. $\beta$-actin was used as the loading control.

and pGSK $3 \beta^{\text {Ser9 }}$ were increased in $P D K 1 \mathrm{cKO}$ mice either at 3 weeks (pGSK3 $\alpha$ : control $=100 \pm 6.3 \%$, cKO $=244.8 \pm 15.1 \%$; pGSK3 $\beta$ : control $=100 \pm 11.2 \%, \mathrm{cKO}=187.3 \pm 13.7 \%$; ps $<0.01$ ) or 3 months (pGSK3 $\alpha$ : control $=100 \pm 1.6 \%$, $\mathrm{cKO}=146.6 \pm 5.3 \%$; pGSK3 $\beta$ : control $=100 \pm 8.0 \%$, $\mathrm{cKO}=148.5 \pm 7.4 \% ;$ ps $<0.01 ; n=3-4$ /group/age) . Due to negative correlation between pGSK $3 \alpha^{\operatorname{Ser} 21} / 3 \beta^{\operatorname{Ser} 9}$ levels and GSK3 activity, this finding suggested that GSK3 was inactivated. In agreement with this, increased $\mathrm{pGSK} 3 \alpha^{\mathrm{Ser} 21} / 3 \beta^{\operatorname{Ser} 9}$ was reported in $P D K 1^{f / f}$;GFAP-Cre mice as well (Figure 4 in Chalhoub et al., 2009).

It has been shown that GSK3 is phosphorylated by several kinases including Akt (Cross et al., 1995) and PKA (Fang et al., 2000; Li et al., 2000). First, Western analysis on pAkt substrates was performed to examine overall Akt activity. Our results showed that levels for several bands of Akt substrates were reduced in $P D K 1 \mathrm{cKO}$ mice as compared to controls (Figure 7A), suggesting decreased activity. Second, Western analysis on pPKA substrates was performed. A number of pPKA substrates exhibited increased levels (Figure 7B), suggesting enhanced activity. Consistent with this, relative levels for $\mathrm{pCREB}^{\mathrm{Ser} 133}$, a well-known PKA substrate, were increased in PDK1 cKO mice (Figure 7B: control $=100 \pm 5.8 \%$; $\mathrm{cKO}=387.1 \pm 37.2 \%$; $p<0.01)$. In contrast, levels of PKA regulatory subunit $1 \alpha$ (PKA Reg $1 \alpha$ ) were not changed (Figure 7B: control $=100 \pm 1.2 \%$; $\mathrm{cKO}=99.1 \pm 7.2 \% ; p>0.9)$.

Previous evidence has demonstrated that the mTOR signaling is critical for neuronal survival (Sarbassov et al., 2005; Kim et al., 2010) and dendritic morphology (Jaworski et al., 2005; Kumar et al., 2005). To investigate whether mTORC1 activity was affected, we examined pS6K and pS6 using cortical samples at 3 months. We found that levels for total $\mathrm{S} 6 \mathrm{~K}$ and $\mathrm{S} 6$ were not changed in $P D K 1 \mathrm{cKO}$ mice (Figures $7 \mathrm{C}, \mathbf{D}$,

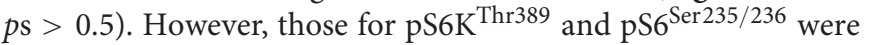
dramatically decreased $(p s<0.05)$. Overall, mTORC1 activity was decreased in PDK1 cKO mice.

\section{DISCUSSION}

PDK1 is a key member in the PI3K signaling (Mora et al., 2004; Engelman et al., 2006) and has been implicated in neurological diseases (Liu et al., 2011; Pietri et al., 2013). Early embryonic lethality of $P D K 1^{-/-}$mice excludes the possibility to study whether loss of endogenous PDK1 affects neuronal survival during cortical development. In this study, viable forebrainspecific PDK1 cKO mice were generated. The following novel findings were reported. First, conditional deletion of PDK1 in the forebrain causes dramatic neuron loss and increased apoptotic cell death. Second, conditional deletion of PDK1 in the forebrain results in impaired mTOR activity.

Microcephaly has been observed in early work using PDK1 cKO line in which PDK1 is conditionally inactivated in neurons and astrocytes of the whole brain (Chalhoub et al., 2009). Given that PDK1 is specifically deleted in the forebrain of $P D K 1^{f / f}$;Emx1-Cre mice, our findings are somehow consistent with those reported previously (Chalhoub et al., 2009; Itoh et al., 2016). First, the current model differs from the line Chalhoub et al. (2009), in that the phenotype in the cerebellum is not the same. This is likely due to that the inactivation of PDK1 
does not occur in neurons of the cerebellum in our cKO line. Second, unlike PDK1 ${ }^{f / f}$;GFAP-Cre (Chalhoub et al., 2009) or $P D K 1^{f / f} ; E m \times 1$-Cre (this study), PDK1 $1^{f / f}$;Nestin-Cre mice die shortly after birth (Itoh et al., 2016) and therefore cannot be used for the study on postnatal brain. Third, PDK1 $1^{f / f} ;$ Nex-Cre mice survive to adulthood and display cortical lamination defect (Itoh et al., 2016). Moreover, it has been nicely demonstrated that abnormal cortical lamination in $P D K 1^{f / f} ; \mathrm{Nex}$-Cre mice is caused by impairment on PDK1/Akt-dependent neuronal migration (Itoh et al., 2016).

Since the total number of NeuN+ cells is dramatically reduced and the length of dendrites is significantly decreased in PDK1 cKO mice, these could directly lead to the formation of small cortex. Overall, endogenous PDK1 may negatively regulate apoptosis in the cortex and therefore control the brain/cortex size. Interestingly, apoptosis is involved in cell death in brain diseases displaying age-related neuron loss. First, previous studies have shown that increased apoptosis is associated with neuron loss in neurodegenerative mouse models (Feng et al., 2004; Tabuchi et al., 2009; Wines-Samuelson et al., 2010; Cheng et al., 2015). Second, early work has demonstrated increased apoptotic cell death in the brain of AD (Su et al., 1994; Lassmann et al., 1995; Anderson et al., 1996).

Since increased number of TUNEL+/NeuN+ or TUNEL+/Tuj1+ cells was found in PDK1 cKO mice, it was reasonable to conclude that neuronal apoptosis contributes to cortical neuron loss. In contrast, neuron loss in PDK1 cKO mice is unlikely due to proliferation of NPCs during development, since our results showed that the self-renewal of NPCs was not impaired as revealed by the BrdU pulse-labeling and PH3 IHC experiments.

To explore the underlying molecular mechanisms, we focused on the Akt/mTOR pathway. First of all, our biochemical results on $\mathrm{pAkt}^{\mathrm{Thr} 308}$ and pAkt substrates have strongly suggested that conditional inactivation of PDK1 leads to reduced Akt activity. Second, our analyses on pS6K and pS6 indicated decreased activity of mTORC1 in PDK1 cKO mice. Third, $P D K 1^{f / f}$;Emx1-Cre mice exhibit increased pGSK3 levels, which may be caused by enhanced PKA activity. Consistent with this notion, previous evidence has shown that PKA inhibits GSK3 by phosphorylating the Ser21/Ser9 of GSK3 $\alpha / 3 \beta$ (Fang et al., 2000), and that the phosphorylation of GSK3 by PKA does not require activation of Akt ( $\mathrm{Li}$ et al., 2000). Overall, since PDK1, Akt, or mTOR is important for cell/neuron survival and dendritic morphogenesis (Datta et al., 1997;

\section{REFERENCES}

Alessi, D. R., James, S. R., Downes, C. P., Holmes, A. B., Gaffney, P. R. J., Reese, C. B., et al. (1997). Characterization of a 3-phosphoinositidedependent protein kinase which phosphorylates and activates protein kinase B. Curr. Biol. 7, 261-269. doi: 10.1016/S0960-9822(06) 00122-9

Anderson, A. J., Su, J. H., and Cotman, C. W. (1996). DNA damage and apoptosis in Alzheimer's disease: colocalization with c- Jun immunoreactivity, relationship to brain area, and effect of postmortem delay. J. Neurosci. 16, 1710-1719.
Jaworski et al., 2005; Kumar et al., 2005; Sarbassov et al., 2005; Kharebava et al., 2008; Kim et al., 2010), it is reasonable to conclude that PDK1 may control neuronal survival during cortical development via the activation of $\mathrm{Akt} / \mathrm{mTOR}$ signaling.

In this study, we report that $P D K 1 \mathrm{cKO}$ mice display deficit on spatial learning and memory. Since it has been demonstrated that neuron loss and synaptic loss directly contribute to cognitive impairments in neurodegenerative disease (GomezIsla et al., 1997; Terry, 2000), we reason that learning deficit in PDK1 cKO mice is likely caused by massive loss of neurons and synapses in the cortex. However, since there was an increase in open-field activity in PDK1 cKO mice, it cannot be ruled out that increased anxiety may directly cause learning impairment or exacerbate learning deficit in PDK1 mutant mice.

\section{AUTHOR CONTRIBUTIONS}

CX, LY, JH, RJ, HW, CH, TL, QW, and XZ conducted experiments. GC, YX, ZYY, and ZZY designed experiments. GC, ZYY, CX, and LY analyzed the data. GC, TS-J, and RM wrote the article.

\section{FUNDING}

This work was supported by grants from the National Basic Research Program of Ministry of Science and Technology of China (2014CB942804), the National Natural Science Foundation of China (31271123, 81230026, and 81630028), the National Key Research and Development Program of China (2016YFC1300504), the Key Research and Development Program of Nanjing Municipal Health Bureau (ZKX16031), the Fundamental Research Funds for the Central Universities (14380012 and 14380322), the Jiangsu Provincial Key Medical Discipline (ZDXKA2016020), and Alzheimer's Research in the United Kingdom.

\section{SUPPLEMENTARY MATERIAL}

The Supplementary Material for this article can be found online at: https://www.frontiersin.org/articles/10.3389/fncel. 2017.00330/full\#supplementary-material

Calleja, V., Alcor, D., Laguerre, M., Park, J., Vojnovic, B., Hemmings, B. A., et al. (2007). Intramolecular and intermolecular interactions of protein kinase B define its activation in vivo. PLOS Biol. 5:e95. doi: 10.1371/journal.pbio. 0050095

Chalhoub, N., Zhu, G., Zhu, X., and Baker, S. J. (2009). Cell type specificity of PI3K signaling in Pdk1- and Pten-deficient brains. Genes Dev. 23, 1619-1624. doi: 10.1101/gad.1799609

Chen, G., Zou, X., Watanabe, H., van Deursen, J. M., and Shen, J. (2010). CREB binding protein is required for both short-term and long-term memory formation. J. Neurosci. 30, 13066-13077. doi: 10.1523/JNEUROSCI.2378-10. 2010 
Cheng, S., Hou, J., Zhang, C., Xu, C., Wang, L., Zou, X., et al. (2015). Minocycline reduces neuroinflammation but does not ameliorate neuron loss in a mouse model of neurodegeneration. Sci. Rep. 5:10535. doi: 10.1038/srep10535

Cordon-Barris, L., Pascual-Guiral, S., Yang, S., Gimenez-Llort, L., LopePiedrafita, S., Niemeyer, C., et al. (2016). Mutation of the 3-phosphoinositidedependent protein kinase 1 (PDK1) substrate-docking site in the developing brain causes microcephaly with abnormal brain morphogenesis independently of Akt, leading to impaired cognition and disruptive behaviors. Mol. Cell. Biol. 36, 2967-2982. doi: 10.1128/MCB.00230-16

Cross, D. A., Alessi, D. R., Cohen, P., Andjelkovich, M., and Hemmings, B. A. (1995). Inhibition of glycogen synthase kinase- 3 by insulin mediated by protein kinase B. Nature 378, 785-789. doi: 10.1038/378785a0

Dainichi, T., Hayden, M. S., Park, S.-G., Oh, H., Seeley, J. J., Grinberg-Bleyer, Y., et al. (2016). PDK1 is a regulator of epidermal differentiation that activates and organizes asymmetric cell division. Cell Rep. 15, 1615-1623. doi: 10.1016/j. celrep.2016.04.051

Datta, S. R., Dudek, H., Tao, X., Masters, S., Fu, H., Gotoh, Y., et al. (1997). Akt phosphorylation of BAD couples survival signals to the cell-intrinsic death machinery. Cell 91, 231-241. doi: 10.1016/S0092-8674(00)80405-5

Engelman, J. A., Luo, J., and Cantley, L. C. (2006). The evolution of phosphatidylinositol 3-kinases as regulators of growth and metabolism. Nat. Rev. Genet. 7, 606-619. doi: 10.1038/nrg1879

Fang, X., Yu, S. X., Lu, Y., Bast, R. C., Woodgett, J. R., and Mills, G. B. (2000). Phosphorylation and inactivation of glycogen synthase kinase 3 by protein kinase A. Proc. Natl. Acad. Sci. U.S.A. 97, 11960-11965. doi: 10.1073/pnas. 220413597

Feng, Q., Di, R., Tao, F., Chang, Z., Lu, S., Fan, W., et al. (2010). PDK1 regulates vascular remodeling and promotes epithelial-mesenchymal transition in cardiac development. Mol. Cell. Biol. 30, 3711-3721. doi: 10.1128/MCB. 00420- 10

Feng, R., Wang, H., Wang, J., Shrom, D., Zeng, X., and Tsien, J. Z. (2004). Forebrain degeneration and ventricle enlargement caused by double knockout of Alzheimer's presenilin-1 and presenilin-2. Proc. Natl. Acad. Sci. U.S.A. 101, 8162-8167. doi: 10.1073/pnas.0402733101

Gomez-Isla, T., Hollister, R., West, H., Mui, S., Growdon, J. H., Petersen, R. C., et al. (1997). Neuronal loss correlates with but exceeds neurofibrillary tangles in Alzheimer's disease. Ann. Neurol. 41, 17-24. doi: 10.1002/ana.410410106

Gorski, J. A., Talley, T., Qiu, M., Puelles, L., Rubenstein, J. L., and Jones, K. R. (2002). Cortical excitatory neurons and glia, but not GABAergic neurons, are produced in the Emx1-expressing lineage. J. Neurosci. 22, 6309-6314.

Itoh, Y., Higuchi, M., Oishi, K., Kishi, Y., Okazaki, T., Sakai, H., et al. (2016). PDK1Akt pathway regulates radial neuronal migration and microtubules in the developing mouse neocortex. Proc. Natl. Acad. Sci. U.S.A. 113, E2955-E2964. doi: $10.1073 /$ pnas. 1516321113

Jaworski, J., Spangler, S., Seeburg, D. P., Hoogenraad, C. C., and Sheng, M. (2005). Control of dendritic arborization by the phosphoinositide-3'-kinaseAkt-mammalian target of rapamycin pathway. J. Neurosci. 25, 11300-11312. doi: 10.1523/JNEUROSCI.2270-05.2005

Kharebava, G., Makonchuk, D., Kalita, K. B., Zheng, J.-J., and Hetman, M. (2008). Requirement of 3-phosphoinositide-dependent protein kinase-1 for BDNF-mediated neuronal survival. J. Neurosci. 28, 11409-11420. doi: 10.1523/ JNEUROSCI.2135-08.2008

Kim, S., Lehtinen, M. K., Sessa, A., Zappaterra, M. W., Cho, S.-H., Gonzalez, D., et al. (2010). The apical complex couples cell fate and cell survival to cerebral cortical development. Neuron 66, 69-84. doi: 10.1016/j.neuron.2010.03.019

Kumar, V., Zhang, M. X., Swank, M. W., Kunz, J., and Wu, G. Y. (2005). Regulation of dendritic morphogenesis by Ras-PI3K-Akt mTOR and Ras-MAPK signaling pathways. J. Neurosci. 25, 11288-11299. doi: 10.1523/JNEUROSCI.2284-05. 2005

Lassmann, H., Bancher, C., Breitschopf, H., Wegiel, J., Bobinski, M., Jellinger, K., et al. (1995). Cell death in Alzheimer's disease evaluated by DNA fragmentation in situ. Acta Neuropathol. 89, 35-41. doi: 10.1007/BF00294257

Lawlor, M. A., Mora, A., Ashby, P. R., Williams, M. R., Murray-Tait, V., Malone, L., et al. (2002). Essential role of PDK1 in regulating cell size and development in mice. EMBO J. 21, 3728-3738. doi: 10.1093/emboj/cdf387
Li, M., Wang, X., Meintzer, M. K., Laessig, T., Birnbaum, M. J., and Heidenreich, K. A. (2000). Cyclic AMP promotes neuronal survival by phosphorylation of glycogen synthase kinase 3ß. Mol. Cell. Biol. 20, 9356-9363. doi: 10.1128/MCB. 20.24.9356-9363.2000

Liu, Y., Liu, F., Grundke-Iqbal, I., Iqbal, K., and Gong, C. X. (2011). Deficient brain insulin signalling pathway in Alzheimer's disease and diabetes. J. Pathol. 225, 54-62. doi: 10.1002/path.2912

Mora, A., Komander, D., van Aalten, D. M., and Alessi, D. R. (2004). PDK1, the master regulator of AGC kinase signal transduction. Semin. Cell Dev. Biol. 15, 161-170. doi: 10.1016/j.semcdb.2003.12.022

Muzumdar, M. D., Tasic, B., Miyamichi, K., Li, L., and Luo, L. (2007). A global double-fluorescent Cre reporter mouse. Genesis 45, 593-605. doi: 10.1002/dvg. 20335

Pietri, M., Dakowski, C., Hannaoui, S., Alleaume-Butaux, A., Hernandez-Rapp, J., Ragagnin, A., et al. (2013). PDK1 decreases TACE-mediated alpha-secretase activity and promotes disease progression in prion and Alzheimer's diseases. Nat. Med. 19, 1124-1131. doi: 10.1038/nm.3302

Sarbassov, D. D., Ali, S. M., and Sabatini, D. M. (2005). Growing roles for the mTOR pathway. Curr. Opin. Cell Biol. 17, 596-603. doi: 10.1016/j.ceb.2005.09.009

Saura, C. A., Choi, S.-Y., Beglopoulos, V., Malkani, S., Zhang, D., Rao, B. S., et al. (2004). Loss of presenilin function causes impairments of memory and synaptic plasticity followed by age-dependent neurodegeneration. Neuron 42, 23-36. doi: 10.1016/S0896-6273(04)00182-5

Steen, E., Terry, B. M., Rivera, E. J., Cannon, J. L., Neely, T. R., Tavares, R., et al. (2005). Impaired insulin and insulin-like growth factor expression and signaling mechanisms in Alzheimer's disease - is this type 3 diabetes. J Alzheimers Dis. 7, 63-80. doi: 10.3233/JAD-20057107

Su, J. H., Anderson, A. J., Cummings, B. J., and Cotman, C. W. (1994). Immunohistochemical evidence for apoptosis in Alzheimer's disease. Neuroreport 5, 2529-2533. doi: 10.1097/00001756-19941200000031

Tabuchi, K., Chen, G., Südhof, T. C., and Shen, J. (2009). Conditional forebrain inactivation of nicastrin causes progressive memory impairment and age-related neurodegeneration. J. Neurosci. 29, 7290-7301. doi: 10.1523/ JNEUROSCI.1320-09.2009

Talbot, K., Wang, H. Y., Kazi, H., Han, L. Y., Bakshi, K. P., Stucky, A., et al. (2012). Demonstrated brain insulin resistance in Alzheimer's disease patients is associated with IGF-1 resistance, IRS-1 dysregulation, and cognitive decline. J. Clin. Invest. 122, 1316-1338. doi: 10.1172/JCI59903

Terry, R. D. (2000). Cell death or synaptic loss in Alzheimer disease. J. Neuropathol. Exp. Neurol. 59, 1118-1119. doi: 10.1093/jnen/59.12.1118

Watatani, K., Hirabayashi, Y., Itoh, Y., and Gotoh, Y. (2012). PDK1 regulates the generation of oligodendrocyte precursor cells at an early stage of mouse telencephalic development. Genes Cells 17, 326-335. doi: 10.1111/j.1365-2443. 2012.01591.x

West, M. J., and Gundersen, H. J. (1990). Unbiased stereological estimation of the number of neurons in the human hippocampus. J. Comp. Neurol. 296, 1-22. doi: $10.1002 /$ cne. 902960102

Wines-Samuelson, M., Schulte, E. C., Smith, M. J., Aoki, C., Liu, X., Kelleher, R. J., et al. (2010). Characterization of age-dependent and progressive cortical neuronal degeneration in presenilin conditional mutant mice. PLOS ONE 5:e10195. doi: 10.1371/journal.pone.0010195

Conflict of Interest Statement: The authors declare that the research was conducted in the absence of any commercial or financial relationships that could be construed as a potential conflict of interest.

Copyright (c) 2017 Xu, Yu, Hou, Jackson, Wang, Huang, Liu, Wang, Zou, Morris, Spires-Jones, Yang, Yin, Xu and Chen. This is an open-access article distributed under the terms of the Creative Commons Attribution License (CC BY). The use, distribution or reproduction in other forums is permitted, provided the original author(s) or licensor are credited and that the original publication in this journal is cited, in accordance with accepted academic practice. No use, distribution or reproduction is permitted which does not comply with these terms. 